\title{
Potential of Endophytic Diaporthe sp. as a New Source of Bioactive Compounds
}

\author{
Kashvintha Nagarajan ${ }^{1}$, Woei-Yenn Tong ${ }^{2}$, Chean-Ring Leong ${ }^{2}$, and Wen-Nee Tan ${ }^{1 *}$ \\ ${ }^{1}$ Chemistry Section, School of Distance Education, Universiti Sains Malaysia, 11800 Minden, Penang, Malaysia \\ ${ }^{2}$ Drug Discovery and Delivery Research Laboratory, Universiti Kuala Lumpur, Malaysian Institute of Chemical and \\ Bioengineering Technology, 78000 Alor Gajah, Melaka, Malaysia
}

\begin{abstract}
Endophytic fungi are symbiotically related to plants and spend most of their life cycle within them. In nature, they have a crucial role in plant micro-ecosystem. They are harnessed for their bioactive compounds to counter human health problems and diseases. Endophytic Diaporthe sp. is a widely distributed fungal genus that has garnered much interest within the scientific community. A substantial number of secondary metabolites have been detected from Diaporthe sp. inhabited in various plants. As such, this minireview highlights the potential of Diaporthe sp. as a rich source of bioactive compounds by emphasizing on their diverse chemical entities and potent biological properties. The bioactive compounds produced are of significant importance to act as new lead compounds for drug discovery and development.
\end{abstract}

Keywords: Endophytes, Diaporthe sp., Phomopsis sp., bioactive compounds, bioactive natural products, drug discovery

\section{Introduction}

Endophytic fungi are microorganisms that inhabit the living tissues of plants without posing any harmful or deleterious symptoms to them. Often, plants accommodate one or many endophytic fungi [1]. The interaction between endophytic fungi and their hosts is mutual, as the hosts supply habitation and nutrients while the fungi secrete functional metabolites for plant growth and survival [2]. Metabolites produced by endophytic fungi have been shown to possess structural diversity and exhibited a broad range of biological activities $[1,3]$. These metabolites, at some point, are similar to those produced by the host plants; indicating the potential of endophytic fungi as an alternative source of bioactive compounds [1]. Hence, it is not surprising that endophytic fungi have attracted considerable attention in producing novel bioactive compounds for exploitation in medicine, agriculture and modern industries [4].

Among the vast endophytic fungi, genus Diaporthe (anamorph of Phomopsis) is known for its strong biosynthetic capability to produce bioactive metabolites $[3,5]$. Diaporthe $\mathrm{sp}$. is a widely distributed fungal genus and colonizes a broad range of hosts. It consists of approximately 800 species, with more than 950 species attributed to Phomopsis sp. [6]. It is commonly isolated from above-ground plants, particularly from tropical and temperate woody plants [7]. Secondary metabolites isolated from Diaporthe sp. have displayed a broad spectrum of biological activities with diverse chemical entities $[8,9]$. As such, this minireview summarizes the bioactive compounds produced from Diaporthe sp. (duration 2015-2020 February) that had colonized within different host plants, particularly highlighting on their unique chemical entities. The bioactive compounds discovered in Diaporthe sp. are reported within the interest of biological context, and thereby, their potential as therapeutic agents.

Received: May 6, 2020 Accepted: June 22, 2020

First published online: June 24,2020

*Corresponding author Phone: +604-6534563 Fax: $+604-6576000$ E-mail: tanwn@usm.my pISSN 1017-7825 eISSN 1738-8872

Copyright(C) 2021 by The Korean Society for Microbiology and Biotechnology

\section{Bioactive compounds from Diaporthe sp.}

Plant-derived fungi of Diaporthe have been reported to produce numerous types of compounds that exhibit a range of biological activities. These compounds offer abundant bioactive core skeletons for new medicinal lead compounds, thus contributing to the research of drug discovery and development. The unique classes of compounds are discussed in the following section. Table 1 lists the isolated compounds from Diaporthe sp.

\section{Mycoepoxydienes}

Fungal mycoepoxydienes were among the bioactive compounds isolated from Diaporthe sp.. They are formed via polyketide pathway through the condensation of acetyl-coenzyme A (CoA) and malonyl-CoA [10]. Mycoepoxydienes have a rare oxygen-bridged cyclooctadiene that serves as the core with $\alpha, \beta$-unsaturated lactone moiety [11]. In 2015, Mandavid et al. reported the isolation of mycoepoxydiene (1) from the ethyl acetate extract of Diaporthe sp. SNB-GSS10. The compound exhibited potent cytotoxicity against human uterine cervical carcinoma $\mathrm{KB}$, human breast cancer MDA-MB- 435 and human lung fibroblast MRC-5 cells with $\mathrm{IC}_{50} 7.5,17.7$ and 


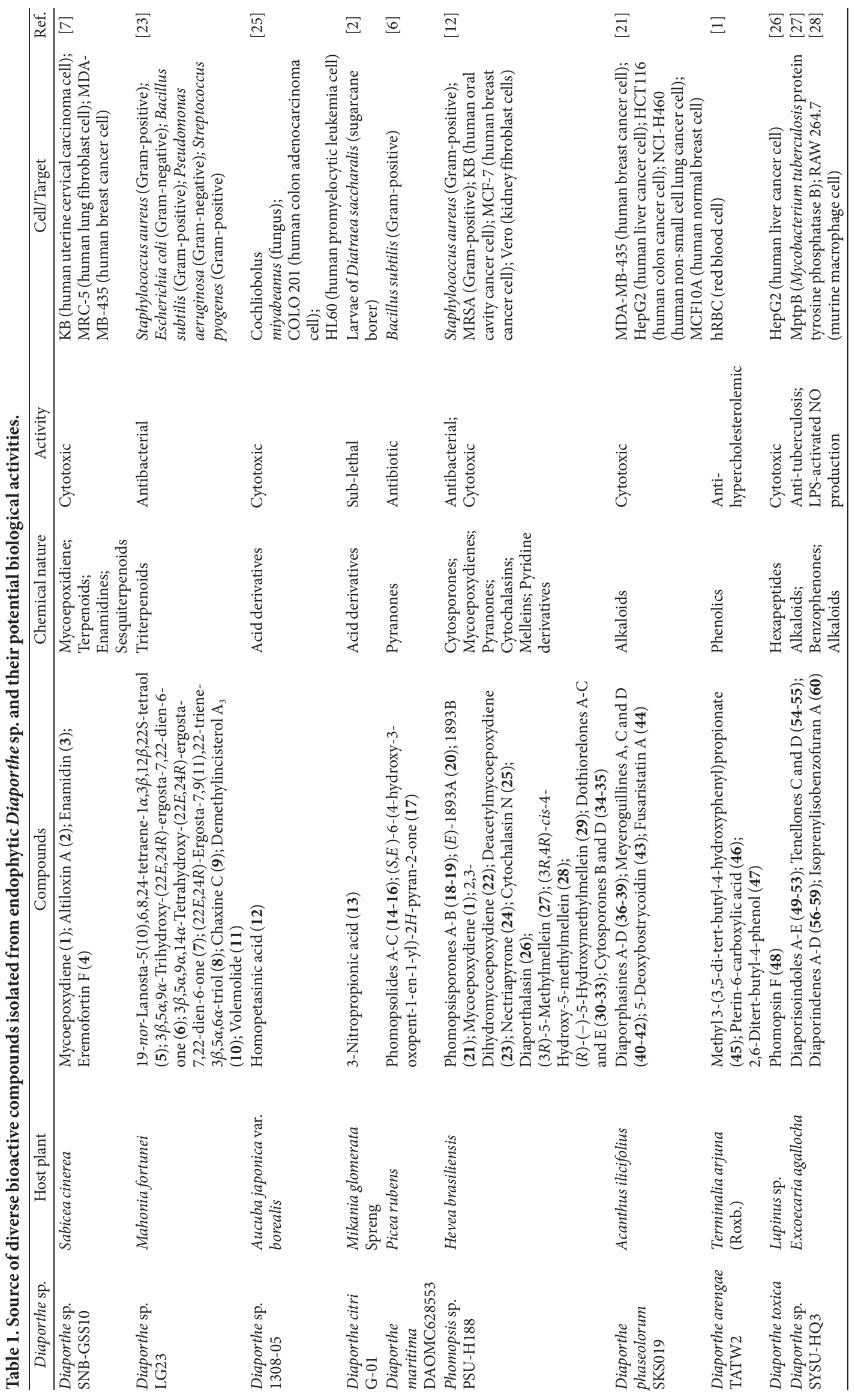




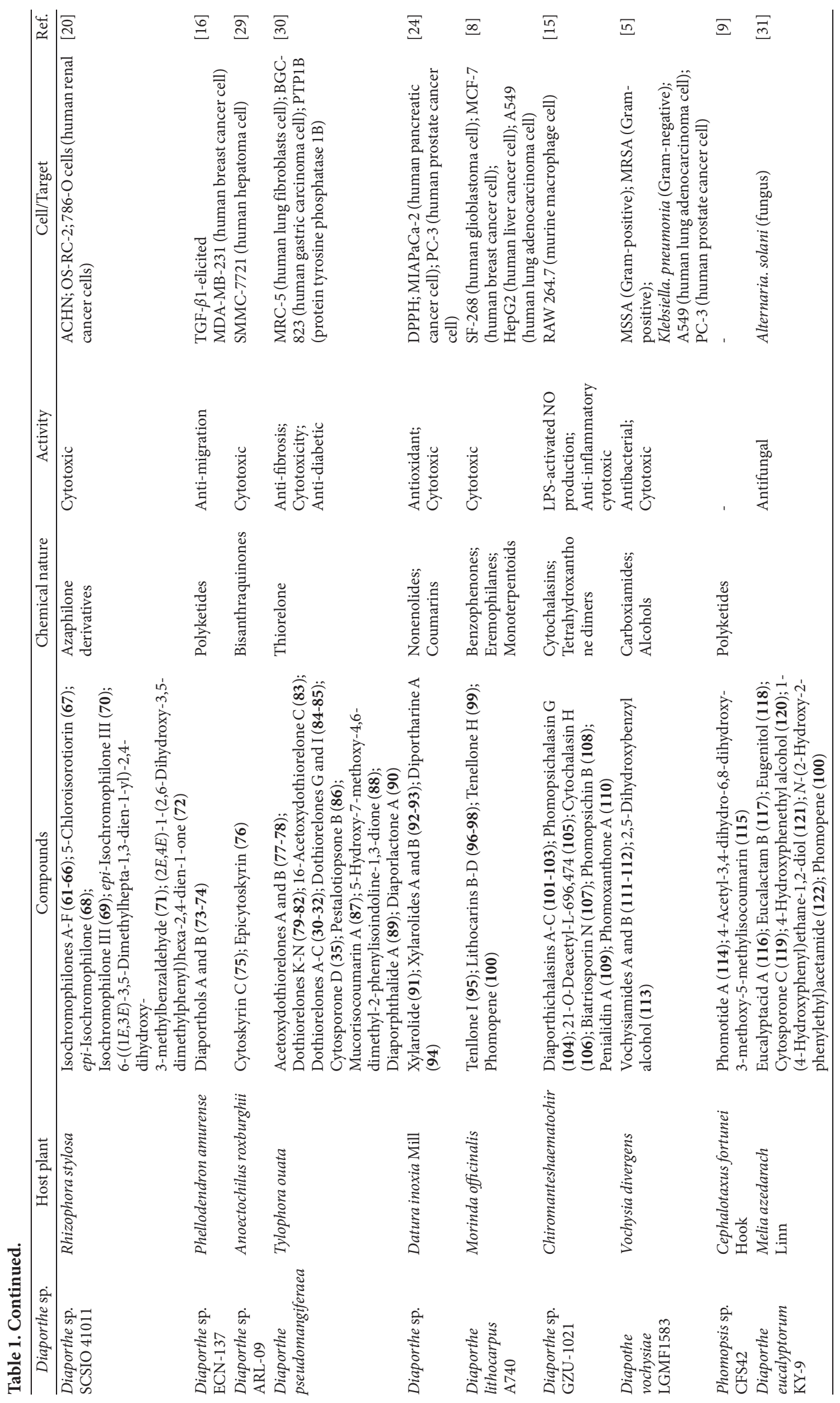




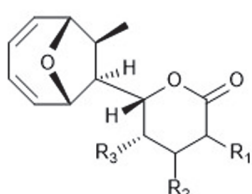<smiles>CC1(C)CCC[C@@]2(C)[C@@H]1C[C@@](C)(O)[C@@H]2C(=O)O</smiles><smiles>CC(C)C(O)C(=O)N/C=C\C(=O)O</smiles>

(3)<smiles>C=C(C)C1CC2(CO)C(=CC1=O)CCC(OC(=O)C(C)C(O)/C=C/C=C/CC(C)O)[C@H]2C</smiles>

(4)

(1) $\mathrm{R}_{1}+\mathrm{R}_{2}=$ double bond; $\mathrm{R}_{3}=\mathrm{O}(\mathrm{CO}) \mathrm{CH}_{3}$

(22) $\mathrm{R}_{1}=\mathrm{R}_{2}=\mathrm{H} ; \mathrm{R}_{3}=\mathrm{O}(\mathrm{CO}) \mathrm{CH}_{3}$

(23) $\mathrm{R}_{1}+\mathrm{R}_{2}=$ double bond; $\mathrm{R}_{3}=\mathrm{OH}$

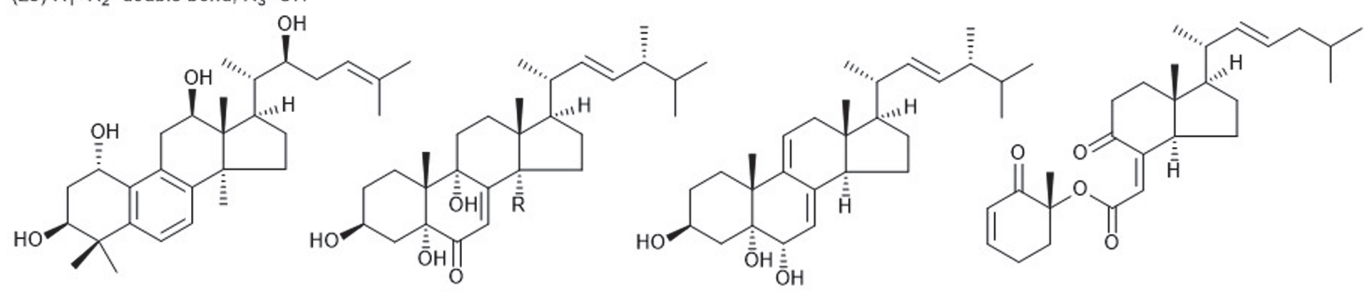

(5)

(6) $\mathrm{R}=\mathrm{H}$<smiles>[R6]C12CC[C@@]3(C)[C@@H](CC[C@@H]3[C@H](C)/C=C/[C@H](C)C=C)C1=CC(=O)O2</smiles>

(7) $\mathrm{R}=\mathrm{OH}$

(8)

(9)

$$
\begin{aligned}
& \text { (10) } \mathrm{R}=\mathrm{H} \\
& \text { (11) } \mathrm{R}=\mathrm{OH}
\end{aligned}
$$<smiles>C=C(C)[C@H]1C[C@@]2(C)C(=CC1=O)CC[C@@H](OC(=O)[C@H](C)[C@H](O)/C=C/C=C/C(=O)CO)[C@@H]2C</smiles>

(12)<smiles>C/C=C(\C)C(=O)O[C@H]1C=CC(=O)O[C@@H]1/C=C/C(=O)[C@@H](C)O</smiles>

(13)
(14)<smiles>[R]C[C@H]([R2])[C@H]([R3])CCCCC(=O)c1c(O)cc(O)cc1CC(=O)O[R]</smiles>

(17)

(18) $\mathrm{R}_{1}=\mathrm{O}(\mathrm{CO}) \mathrm{CH}_{2} \mathrm{CH}_{2} \mathrm{NO}_{2} ; \mathrm{R}_{2}=\mathrm{R}_{3}=\mathrm{H} ; \mathrm{R}_{4}=\mathrm{CH}_{2} \mathrm{CH}_{3}$ (19) $\mathrm{R}_{1}=\mathrm{O}(\mathrm{CO}) \mathrm{CH}_{3} ; \mathrm{R}_{2}=\mathrm{R}_{3}=\mathrm{H}_{;} ; \mathrm{R}_{4}=\mathrm{CH}_{2} \mathrm{CH}_{3}$

(30) $\mathrm{R}_{1}=\mathrm{R}_{3}=\mathrm{H} ; \mathrm{R}_{2}=\mathrm{OH} ; \mathrm{R}_{4}=\mathrm{CH}_{2} \mathrm{CH}_{3}$

(31) $\mathrm{R}_{1}=\mathrm{R}_{2}=\mathrm{H} ; \mathrm{R}_{3}=\mathrm{OH} ; \mathrm{R}_{4}=\mathrm{CH}_{2} \mathrm{CH}_{3}$ (32) $\mathrm{R}_{1}=\mathrm{OH} ; \mathrm{R}_{2}=\mathrm{R}_{3}=\mathrm{H} ; \mathrm{R}_{4}=\mathrm{CH}_{2} \mathrm{CH}_{3}$

(33) $\mathrm{R}_{1}=\mathrm{R}_{2}=\mathrm{R}_{3}=\mathrm{H}_{;} \mathrm{R}_{4}=\mathrm{CH}_{3}$

(34) $R_{1}=R_{2}=R_{3}=H ; R_{4}=C_{2} C_{3}$

(20)

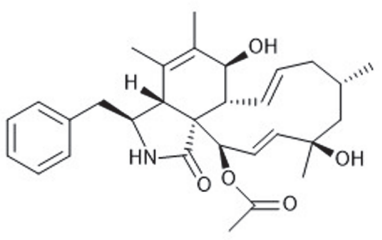

(25)
(21)<smiles></smiles><smiles>CC=C(C)C1=CC(OC)=C2CCCC2C1</smiles>

(24)<smiles>[R8]c1ccc(O)c2c1C([R2])[C@H](C)OC2=O</smiles>

(27) $\mathrm{R}_{1}=\mathrm{CH}_{3} ; \mathrm{R}_{2}=\mathrm{H}$ (28) $\mathrm{R}_{1}=\mathrm{CH}_{3} ; \mathrm{R}_{2}=\mathrm{OH}$ (29) $\mathrm{R}_{1}=\mathrm{CH}_{2} \mathrm{OH} ; \mathrm{R}_{2}=\mathrm{H}$

(26)<smiles>CCCCCCCC1OC(=O)Cc2cc(O)c(O)c(O)c21</smiles>

(35)

Fig. 1A. Bioactive compounds (1-35) isolated from endophytic Diaporthe sp.

$15.8 \mu \mathrm{M}$, respectively [7]. In a study on Phomopsis sp. PSU-H188, five mycoepoxydiene derivatives; (E)-1893A (20), 1893 B (21), mycoepoxydiene (1), 2,3-dihydromycoepoxydiene (22) and deacetylmycoepoxydiene (23) were obtained. Among them, $(E)$-1893A was isolated for the first time in fungal endophytes. Similar to other reports, mycoepoxydiene was found active against human breast cancer MCF-7 ( IC $_{50} 9.27 \mu \mathrm{M}$ ) and human oral cavity cancer $\mathrm{KB}\left(\mathrm{IC}_{50} 14.43 \mu \mathrm{M}\right)$ cells while the rest were less active [7, 12]. Based on the cytotoxic mechanisms of mycoepoxydiene derivatives, they are able to induce cancer cells apoptosis in vivo, thus reflecting their potential for the development of anti-cancer drugs [13].

\section{Cytochalasins}

Cytochalasin, which has often been regarded as mycotoxin, is characterized by a substituted perhydroisoindolone moiety joined to a macrocycle [14]. In 2017, two cytochalasin derivatives, known as cytochalasin N (25) and diaporthalasin (26), were isolated from Phomopsis sp. PSU-H188. Both compounds did not show any cytotoxicity on cancerous KB and MCF-7 cells, but cytochalasin N exerted a significant cytotoxic effect on noncancerous Vero cells at $4.89 \mu \mathrm{M}$. Meanwhile, diaporthalasin was found to inhibit Gram-positive MRSA and 


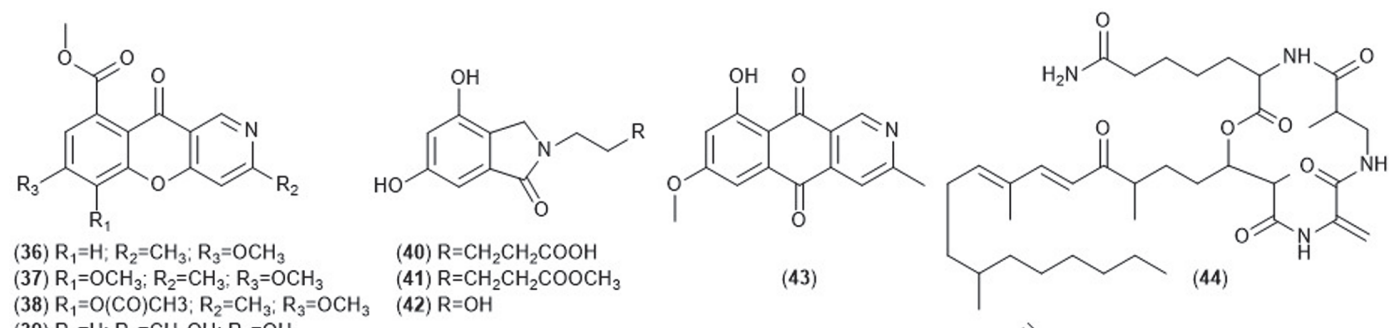
(38) $\mathrm{R}_{1}=\mathrm{O}(\mathrm{CO}) \mathrm{CH} 3 ; \mathrm{R}_{2}=\mathrm{CH}_{3} ; \mathrm{R}_{3}=\mathrm{OCH}$ (39) $\mathrm{R}_{1}=\mathrm{H} ; \mathrm{R}_{2}=\mathrm{CH}_{2} \mathrm{OH} ; \mathrm{R}_{3}=\mathrm{OH}$<smiles>CC(C)(C)c1cc(CCC(=O)O)cc(C(C)(C)C)c1O</smiles>

(45)<smiles>Nc1nc2ncc(C(=O)O)nc2c(=O)[nH]1</smiles>

(46)<smiles>CC(C)(C)c1cccc(C(C)(C)C)c1O</smiles>

(47)

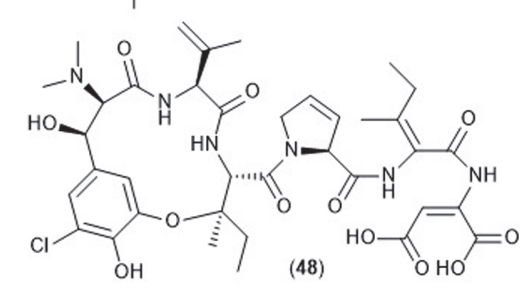<smiles>[R]C1(c2cc(C)cc3c2O[C@H](C(C)(C)O)CO3)NC(=O)c2c(O)ccc(CC=C(C)C)c21</smiles>

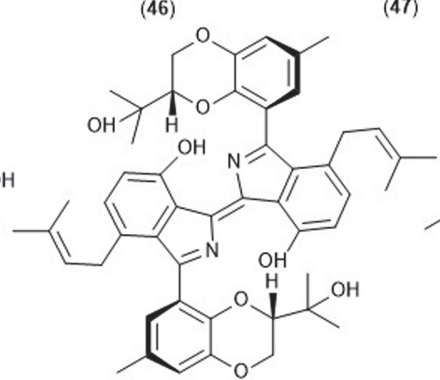

(51)<smiles>[R]OC(=O)c1c(O)ccc(CC=C(C)C)c1C(=O)c1cc(C)cc2c1O[C@H](C(C)(C)O)CO2</smiles>

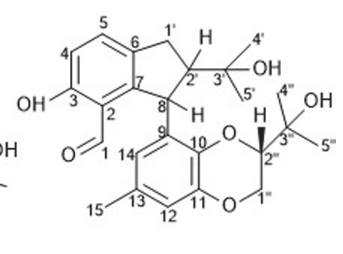

(56) $8 R, 2 ' S, 2 " S$

(57) as, $8 R, 2^{\prime} R, 2$ " $S-2$ (58) aR, $8 S, 2 S, 2$ "S-3 (59) $8 S, 2 ' R, 2 " S$
(52) $\mathrm{R}=\mathrm{OCH}_{3} ; \mathrm{R}$

(53) $\mathrm{R}=\mathrm{OCH}_{3} ; \mathrm{S}$<smiles>CC(C)=CCc1ccc(O)c2c1[C@H](c1cc(C)cc3c1O[C@H](C(C)(C)O)CO3)OC2=O</smiles>

(60)<smiles>[R2]C1([R2])C(Cl)=C2C=C(/C=C/C(C)=C/[C@H](C)CC)OC=C2[C@@]2(C)[C@H](C(C)=O)C(=O)O[C@@]12C</smiles>

(61) $\mathrm{R}_{1}=\mathrm{H} ; \mathrm{R}_{2}=\mathrm{OH} ; 8 \mathrm{~S}, 2$ ' $\mathrm{S}$

(62) $\mathrm{R}_{1}=\mathrm{CH}_{3}, \mathrm{R}_{2}=\mathrm{OCH}_{3} ; 8 \mathrm{~S}, 2$ 'S

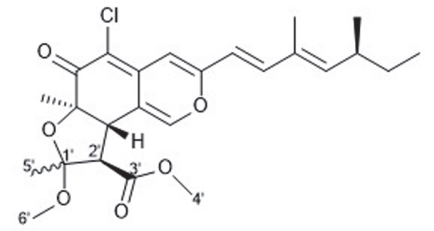

(63) 1 'S<smiles></smiles>

(65) $\mathrm{R}_{1}=\mathrm{R}_{2}=\mathrm{OCH}_{3}$ (67) $\mathrm{R}_{1}+\mathrm{R}_{2}=\mathrm{O}$

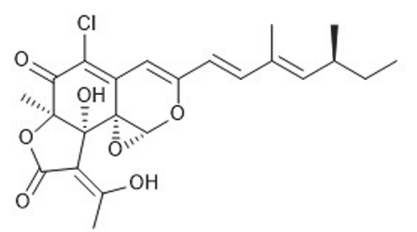

(66)<smiles>CC[C@H](C)/C=C(C)/C=C/C1=CC2=C(Cl)C(=O)[C@](C)(O)[C@H](CC(C)=O)C2CO1</smiles>

(68)

Fig. 1B. Bioactive compounds (36-68) isolated from endophytic Diaporthe sp.

Staphylococcus aureus (MIC $4 \mu \mathrm{g} / \mathrm{ml}$ ), which had no cytotoxicity on non-cancerous cells [12]. In a study that assessed Diaporthe sp. GZU-1021, six cytochalasin derivatives were obtained. Among them, diaporthichalasins A-C (101-103) were identified as new compounds, whereas phomopsichalasin G (104), 21-O-deacetyl-L-696,474 (105) and cytochalasin, H (106) were known compounds. Although the compounds had similar cytochalasin skeleton, they displayed different inhibition on lipopolysaccharide-induced nitric oxide (LPS-induced NO) production. It was deduced that both hydroxyl (C-18) and acetyl (C-21) groups present in cytochalasin $\mathrm{H}$ enhanced its bioactivity [15]. Besides, cytochalasins has been found to play a crucial role in interrupting the formation of filamentous actin. They are able to modify cell motility and morphology, adherence or secretion and drug resistance. These properties are essential for the development of chemotherapeutic agents in drug resistant cancer cells [14]. Therefore, multiple chemical syntheses that focus on the various functional groups present in cytochalasins may aid in the discovery of novel bioactive compounds.

\section{Depsidones}

On the other hand, another class of compounds known as depsidones has been found in Diaporthe sp.. In 2018, the discovery of diaporthols A and B (73-74) produced by Diaporthe sp. ECN-137 was reported by Nakashima et 
<smiles>CCC(C)/C=C(C)/C=C/C1=CC2=C(Cl)C(=O)C(C)(O)[C@@H](O)[C@]2(C)C1</smiles>

69) $\beta-\mathrm{OH}$

(70) $\alpha-\mathrm{OH}$

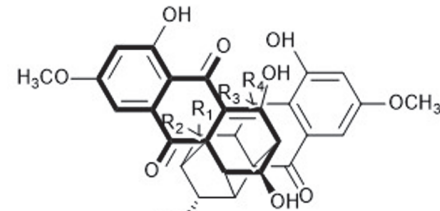

(75) $\mathrm{R}_{1}+\mathrm{R}_{2}=\mathrm{O} ; \mathrm{R}_{3}=\mathrm{H}: \mathrm{R}_{4}=\mathrm{OH}$ (76) $\mathrm{R}_{1}=\mathrm{H} ; \mathrm{R}_{2}=\mathrm{OH} ; \mathrm{R}_{3}+\mathrm{R}_{4}=\mathrm{O}$<smiles>C/C=C/C=C/C(=O)c1c(O)c(C)cc(CCC(C)/C=C/C=C/c2cc(O)c(C)c(O)c2C=O)c1O</smiles>

(71)

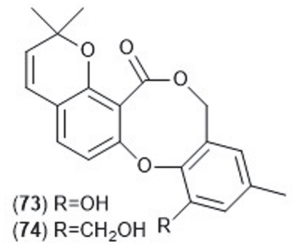

(74) $\mathrm{R}=\mathrm{CH}_{2} \mathrm{O}$

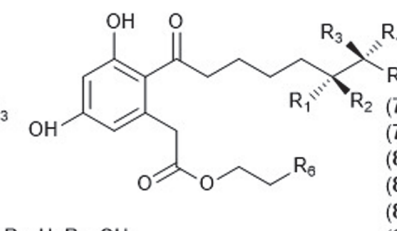

$$
R_{5}
$$

$\mathrm{R}_{4}$

77) $\mathrm{R}_{1}=\mathrm{H} ; \mathrm{R}_{2}=\mathrm{H} ; \mathrm{R}_{3}=\mathrm{OCOCH}_{3}: \mathrm{R}_{4}=\mathrm{H} ; \mathrm{R}_{5}=\mathrm{CH}_{3} ; \mathrm{R}_{6}=\mathrm{H}$ (79) $\mathrm{R}_{1}=\mathrm{H} ; \mathrm{R}_{2}=\mathrm{H} ; \mathrm{R}_{3}=\mathrm{H} ; \mathrm{R}_{4}=\mathrm{H} ; \mathrm{R}_{5}=\mathrm{COOCH}_{3} ; \mathrm{R}_{8}=\mathrm{H}$ (80) $\mathrm{R}_{1}=\mathrm{O} ; \mathrm{R}_{2}=\mathrm{O} ; \mathrm{R}_{3}=\mathrm{H} ; \mathrm{R}_{4}=\mathrm{H} ; \mathrm{R}_{5}=\mathrm{CH}_{3} ; \mathrm{R}_{6}=\mathrm{H}$ (81) $\mathrm{R}_{1}=\mathrm{H} ; \mathrm{R}_{2}=\mathrm{H} ; \mathrm{R}_{3}=\mathrm{H} ; \mathrm{R}_{4}=\mathrm{H} ; \mathrm{R}_{5}=\mathrm{CH}_{3} ; \mathrm{R}_{6}=p-\mathrm{OH}-\mathrm{Ph}$ (82) $\mathrm{R}_{1}=\mathrm{H} ; \mathrm{R}_{2}=\mathrm{H} ; \mathrm{R}_{3}=\mathrm{H} ; \mathrm{R}_{4}=\mathrm{H} ; \mathrm{R}_{5}=\mathrm{CH}_{2} \mathrm{OCOCH}_{3} ; \mathrm{R}_{\mathrm{g}}=p-\mathrm{OH}-\mathrm{Ph}$ (83) $\mathrm{R}_{1}=\mathrm{H} ; \mathrm{R}_{2}=\mathrm{H} ; \mathrm{R}_{3}=\mathrm{H} \cdot \mathrm{R}_{4}=\mathrm{H} ; \mathrm{R}_{5}=\mathrm{CH}_{2} \mathrm{OCOCH}_{3} ; \mathrm{R}_{8}=\mathrm{H}$ (84) $\mathrm{R}_{1}=\mathrm{H} ; \mathrm{R}_{2}=\mathrm{H} ; \mathrm{R}_{3}=\mathrm{H} ; \mathrm{R}_{4}=\mathrm{H} ; \mathrm{R}_{5}=\mathrm{CH}_{3} ; \mathrm{R}_{6}=\mathrm{H}$ (85) $\mathrm{R}_{1}=\mathrm{H} ; \mathrm{R}_{2}=\mathrm{H} ; \mathrm{R}_{3}=\mathrm{O} ; \mathrm{R}_{4}=\mathrm{O} ; \mathrm{R}_{5}=\mathrm{CH}_{3} ; \mathrm{R}_{8}=\mathrm{H}$<smiles>CCOC(=O)Cc1cc(O)cc(O)c1C(=O)CCCCC(CC)OC(C)=O</smiles><smiles>CCCCCCCc1cc(=O)c2c(CC(=O)OCC)cc(O)cc2o1</smiles><smiles>COc1cc(O)c2c(=O)oc(C[C@@H](O)C[C@H](C)O)cc2c1</smiles>

(87)<smiles>COc1c(C)c(O)c(C)c2c1C(=O)N(c1ccccc1)C2=O</smiles>

(88)<smiles>COc1c(C)cc(C(=O)OC[C@H](O)CO)c2c1C(=O)OC2</smiles>

(89)<smiles></smiles>

(91) $\mathrm{R}_{1}=\mathrm{OH} ; \mathrm{R}_{2}=\mathrm{H}$

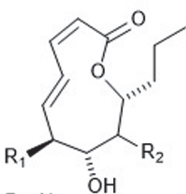

$\mathrm{OH}$

(92) $\mathrm{R}_{1}=\mathrm{OH} ; \mathrm{R}_{2}=\mathrm{CH}\left(\mathrm{CH}_{3}\right) \mathrm{CH}_{2} \mathrm{OCO}-\mathrm{p}-\mathrm{OH}-\mathrm{Ph}$ (93) $\mathrm{R}_{1}=\mathrm{OCH}_{3} ; \mathrm{R}_{2}=\mathrm{H}$<smiles>CCC/C=C/[C@H]1OC(=O)c2cc(O)cc(O)c2[C@H]1O</smiles><smiles>CC(C)=CCc1ccc(O)c2c1C(=O)c1c(c(C)cc3c1O[C@H](C(C)(C)O)CO3)OC2</smiles>

(90)

(95)<smiles>CC(C)=C1C[C@]2(C)C(=CC1=O)CC[C@@H](OC(=O)C(C)C(O)/C=C/C=C/CC(C)O)[C@@H]2C</smiles>

(99)<smiles>[R]C1C[C@]2(C)C(=CC1=O)CC[C@@H](O)[C@@H]2C</smiles>

(96) $\mathrm{R}=\mathrm{C}\left(=\mathrm{CH}_{2}\right) \mathrm{CH}_{3}$ (97) $\mathrm{R}=\left\{=\mathrm{C}\left(\mathrm{CH}_{3}\right)_{2}\right\}$<smiles>CCC(C)C(O)/C=C/C=C/CC(C)OC(C)=O</smiles><smiles>CC(=O)OC1C2CC(C)(OC2(C)C)C1O</smiles>

(98)<smiles>CC(C)(O)[C@H]1CC[C@@](C)(O)[C@H]2O[C@H]12</smiles>

(100)

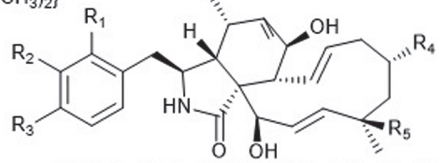

(101) $\mathrm{R}_{1}=\mathrm{H} ; \mathrm{R}_{2}=\mathrm{OH} ; \mathrm{R}_{3}=\mathrm{H} ; \mathrm{R}_{4}=\mathrm{CH}_{3} ; \mathrm{R}_{5}=\mathrm{H}$ (102) $\mathrm{R}_{1}=\mathrm{H} ; \mathrm{R}_{2}=\mathrm{H} ; \mathrm{R}_{3}=\mathrm{OH} ; \mathrm{R}_{4}=\mathrm{CH}_{3} ; \mathrm{R}_{5}=\mathrm{H}$ (103) $\mathrm{R}_{1}=\mathrm{H} ; \mathrm{R}_{2}=\mathrm{H} ; \mathrm{R}_{3}=\mathrm{H} ; \mathrm{R}_{4}=\mathrm{CH}_{2} \mathrm{OH} ; \mathrm{R}_{5}=\mathrm{H}$ (104) $\mathrm{R}_{1}=\mathrm{OH} ; \mathrm{R}_{2}=\mathrm{H} ; \mathrm{R}_{3}=\mathrm{H} ; \mathrm{R}_{4}=\mathrm{CH}_{3} ; \mathrm{R}_{5}=\mathrm{H}$ (105) $\mathrm{R}_{1}=\mathrm{H} ; \mathrm{R}_{2}=\mathrm{H} ; \mathrm{R}_{3}=\mathrm{H} ; \mathrm{R}_{4}=\mathrm{CH}_{3} ; \mathrm{R}_{5}=\mathrm{CH}_{3}$ (106) $\mathrm{R}_{1}=\mathrm{H} ; \mathrm{R}_{2}=\mathrm{H} ; \mathrm{R}_{3}=\mathrm{H} ; \mathrm{R}_{4}=\mathrm{CH}_{3} ; \mathrm{R}_{5}=\mathrm{OH}$

Fig. 1C. Bioactive compounds (69-106) isolated from endophytic Diaporthe sp.

al. The compounds had tetracyclic skeletons, which mimicked the core structure of purpactin A. They were examined for their activity on transforming growth factor- $\beta 1$ (TGF- $\beta 1$ ) induced wound closure of MDA-MB-231 breast cancer cells [16]. The TGF- $\beta 1$, one of the mRNAs detected in most primary breast cancers, has a significant function in apoptosis, angiogenesis and cancer progression [17]. Diaporthols A and B isolated were appeared to suppress the TGF- $\beta 1$-induced wound closure at $20 \mu \mathrm{M}$, hence signifying their potential as tumor inhibitors [16]. Reports have also emphasized that such compounds are active inhibitors in cholesteryl ester transfer protein (CETP) and acyl-CoA:cholesterol acyltransferase (ACAT), which promote the therapeutic potential of atherosclerosis [18]. Therefore, different derivatives of depsidones indicate the potential of developing new drugs.

\section{Azaphilones}

Azaphilones, which consist of a highly oxygenated pyranoquinone bicyclic core skeleton, has received a great deal of scientific interest recently due to their interesting structural features and promising biological activities [19]. In 2018, six new polyoxygenated chloroazaphilones (isochromophilones A-F, (61-66)) and their analogues 
<smiles>CC(O)c1occ2c1C(=O)c1cc(O)cc(O)c1C2=O</smiles>

(107)<smiles>COC(=O)c1cc(OC)c(O)c2oc3c(c(=O)c12)C(OC)O[C@H](C)C3</smiles>

(108)<smiles>CC1(O)Cc2oc3c(C(=O)O)c(O)c(O)cc3c(=O)c2CO1</smiles>

(109)

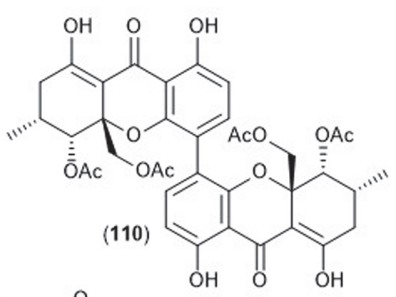<smiles>CC(C)C(O)C(=O)N/C=C\C(=O)O</smiles>

(111)<smiles>CC(=O)C1c2c(C)c(O)cc(O)c2C(=O)OC1C</smiles>

(115)<smiles>COc1cc2oc(C)cc(=O)c2c(O)c1C</smiles>

(118)<smiles>CC(C)C1=CC(=O)OCC(C(C)O)NC1=O</smiles>

(112)<smiles>OCc1cc(O)ccc1O</smiles>

(113)<smiles>CCC(C)C(O)C(C)C=CCC[C@H](O)C[C@@H](O)C(C)C(=O)O</smiles><smiles>CC(=O)[C@H]1c2c(C)c(O)cc(O)c2C(=O)OC1[C@@]1(C(N)=O)OC(C)=CC1=O</smiles>
(114)

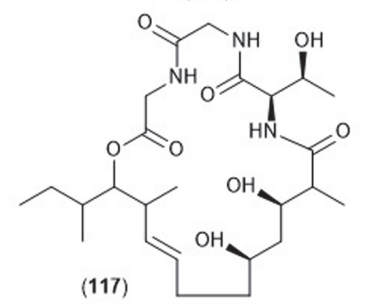

(117)<smiles>CCCCCCC1OC(=O)Cc2ccccc21</smiles>

(119)<smiles>[R]c1ccc(Br)cc1</smiles>

(120) $\mathrm{R}_{1}=\mathrm{OH} ; \mathrm{R}_{2}=\mathrm{CH}_{2} \mathrm{CH}_{2} \mathrm{OH}$

(121) $\mathrm{R}_{1}=\mathrm{OH} ; \mathrm{R}_{2}=\mathrm{CH}(\mathrm{OH}) \mathrm{CH}_{2} \mathrm{OH}$

(122) $\mathrm{R}_{1}=\mathrm{H} ; \mathrm{R}_{2}=\mathrm{CH}(\mathrm{OH}) \mathrm{CH}_{2} \mathrm{NHCOCH}_{3}$

Fig. 1D. Bioactive compounds (107-122) isolated from endophytic Diaporthe sp.

were obtained from the ethyl acetate extract of Diaporthe sp. SCSIO 41011. Among the newly isolated azaphilones, isochromophilones A and B were the first described azaphilones with the absence of a carbonyl group at C-6. Upon being assessed for their cytotoxicity on ACHN, OS-RC-2 and 786-O human renal carcinoma cells; isochromophilone $\mathrm{D}$ displayed cytotoxicity against $786-\mathrm{O}$ cells with the lowest $\mathrm{IC}_{50}(8.9 \mu \mathrm{M})$. In cell cycle arrest and cell apoptosis, the compound induced total apoptotic cells at 56\% (48 h) and $98 \%(72 \mathrm{~h})$ at $10 \mu \mathrm{M} \mathrm{[20].} \mathrm{Apart}$ from this, various bioactivities, such as inhibitions of glycoprotein gp120-CD4 binding, protein Grb2-SH2 and MDM2-p53 interactions, heat shock protein 90 (Hsp90) and dihydrofolate reductase, were reported for azaphilones [19]. Considering the biological diversity of azaphilones, syntheses that alter the side chains or the cyclic moieties should be weighed in to assess their structure-activity relationships, so as to explore their potential for drug discovery.

\section{Other Organic Compounds}

Other metabolites isolated from Diaporthe sp. include alkaloids, terpenoids, pyranones, benzophenones, bisanthraquinones, xanthones, acid derivatives, alcohols and amides. In 2017, Cui and colleagues isolated six new alkaloids, namely diaporphasines A-D (36-39), meyeroguillines $\mathrm{C}$ and D (41-42) along with known meyeroguilline A (40), 5-deoxybostrycoidin (43) and fusaristatin A (44), from D. phaseolorum SKS019. They were described for the first time in Diaporthe sp., as containing the skeletons of chromeno[3,2-c]pyridines and isoindolinones. 5-Deoxybostrycoidin, which was initially isolated from fungus Nectria haematococca, had induced cytotoxicity against human breast cancer MDA-MB-435 and human non-small cell lung cancer NCI$\mathrm{H} 460$ cells with $\mathrm{IC}_{50}$ of 5.32 and $6.57 \mu \mathrm{M}$, respectively $[21,22]$. Meanwhile, a new lanostanoid, known as 19-norlanosta-5(10),6,8,24-tetraene-1 $\alpha, 3 \beta, 12 \beta, 22 S$-tetraol (5) was obtained from Diaporthe sp. LG23. The compound is a rare tetracyclic triterpenoid with an unusual aromatic ring B system, coupled with the loss of a common methyl group at C-19. It exhibited remarkable antibacterial activity on Gram-positive Streptococcus pyogenes at $0.1 \mu \mathrm{g} / \mathrm{ml}$, when compared to gentamicin $(10.0 \mu \mathrm{g} / \mathrm{ml})$ [23]. Additionally, two rare cyclic 10-membered nonenolides, known as xylarolides A and B (92-93), were obtained from Diaporthe sp. isolated from Datura inoxia Mill. It is noteworthy to highlight that xylarolide A showed potent growth inhibition on human pancreatic cancer MIAPaCa-2 and human prostate cancer PC-3 cells with $\mathrm{IC}_{50}$ of 20 and $14 \mu \mathrm{M}$, respectively [24].

Endophytic Diaporthe sp. is an interesting group of microorganisms that provide a rich source of bioactive and chemically diverse compounds with medicinal potential. In this minireview, a range of rare carbon skeletons of compounds isolated from Diaporthe sp. is discussed. They have been reported to possess cytotoxic, antimicrobial, anti-hypercholesterolemic, anti-tuberculosis, anti-fibrosis, anti-diabetic, antioxidant and anti-inflammatory properties. The discovered compounds may act as potential leads for scientists to synthesize potent analogues. It has been observed that most of the bioactivity studies of Diaporthe sp. are focused on in vitro. Therefore, in depth 
in vivo and a series of mechanism studies regarding these bioactive compounds are in need for their future application as therapeutic agents.

\section{Acknowledgments}

This work was supported by the Fundamental Research Grant Scheme (FRGS) (203.PJJAUH.6711662) of Ministry of Higher Education, Malaysia.

\section{Conflict of Interest}

The authors have no financial conflicts of interest to declare.

\section{References}

1. Patil M, Patil R, Mohammad S, Maheshwari V. 2017. Bioactivities of phenolics-rich fraction from Diaporthe arengae TATW2, an endophytic fungus from Terminalia arjuna (Roxb.). Biocatal. Agric. Biotechnol. 10: 396-402.

2. Polonio JC, Ribeiro MAS, Rhoden SA, Sarragiotto MH, Azevedo JL, Pamphile JA. 2016. 3-Nitropropionic acid production by the endophytic Diaporthe citri: molecular taxonomy, chemical characterization, and quantification under pH variation. Fungal Biol. 120: $1600-1608$.

3. Hu Z, Wu Y, Xie S, Sun W, Guo Y, Li XN, et al. 2017. Phomopsterones A and B, two functionalized ergostane-type steroids from the endophytic fungus Phomopsis sp. TJ507A. Org. Lett. 19: 258-261.

4. Xie S, Wu Y, Qiao Y, Guo Y, Wang J, Hu Z, et al. 2018. Protoilludane, illudalane, and botryane sesquiterpenoids from the endophytic fungus Phomopsis sp. TJ507A. J. Nat. Prod. 81:1311-1320.

5. Noriler SA, Savi DC, Ponomareva LV, Rodrigues R, Rohr J, Thorson JS, et al. 2019. Vochysiamides A and B: two new bioactive carboxamides produced by the new species Diaporthe vochysiae. Fitoterapia 138: 104273.

6. Tanney JB, Mcmullin DR, Green BD, Miller JD, Seifert KA. 2016. Production of antifungal and antiinsectan metabolites by the Picea endophyte Diaporthe maritima sp. nov. Fungal Biol. 120: 1448-1457.

7. Mandavid H, Rodrigues AMS, Espindola LS, Eparvier V, Stien D. 2015. Secondary metabolites isolated from the Amazonian endophytic fungus Diaporthe sp. SNB-GSS10. J. Nat. Prod. 78: 1735-1739.

8. Liu H, Chen Y, Li H, Li S, Tan H, Liu Z, et al. 2019. Four new metabolites from the endophytic fungus Diaporthe lithocarpus A740. Fitoterapia 137: 104260

9. Ma K-L, Wei W-J, Li H-Y, Wang L-D, Dong S-H, Gao K. 2020. Phomotide A, a novel polyketide, from the endophytic fungus Phomopsis sp. CFS42. Tetrahedron Lett. 61: 151468.

10. Daley DK, Brown KJ, Badal S. 2017. Fungal Metabolites, pp. 413-421. In Badal S, Delgoda R (eds.), Pharmacognosy, Fundamentals, Applications and Strategies, Academic Press, Elsevier, Massachusetts, USA.

11. Wang J, Zhao B, Zhang W, Wu X, Wang R, Huang Y, et al. 2010. Mycoepoxydiene, a fungal polyketide, induces cell cycle arrest at the G2/M phase and apoptosis in HeLa cells. Bioorg. Med. Chem. Lett. 20: 7054-7058.

12. Kongprapan T, Xu X, Rukachaisirikul V, Phongpaichit S, Sakayaroj J, Chen J, et al. 2017. Cytosporone derivatives from the endophytic fungus Phomopsis sp. PSU-H188. Phytochem. Lett. 22: 219-223.

13. Zhu S, Zhang Y-S, Sheng X-H, Xu M, Wu S-S, Shen Y-M, et al. 2015. Deacetyl-mycoepoxydiene, isolated from plant endophytic fungi Phomosis sp. demonstrates anti-microtubule activity in MCF-7 cells. Biomed. Pharmacother. 69: 82-89.

14. Trendowski M, Christen TD, Acquafondata C, P. Fondy TP. 2015. Effects of cytochalasin congeners, microtubule-directed agents, and doxorubicin alone or in combination against human ovarian carcinoma cell lines in vitro. BMC Cancer 15: 632.

15. Liu Y, Ruan Q, Jiang S, Qu Y, Chen J, Zhao M, et al. 2019. Cytochalasins and polyketides from the fungus Diaporthe sp. GZU-1021 and their anti-inflammatory activity. Fitoterapia 137: 104187.

16. Nakashima K, Tomida J, Kamiya T, Hirai T, Morita Y, Hara H, et al. 2018. Diaporthols A and B: bioactive diphenyl ether derivatives from an endophytic fungus Diaporthe sp. Tetrahedron Lett. 59: 1212-1215.

17. Zarzynska, JM. 2014. Two faces of TGF-Beta1 in breast cancer. Mediators Inflamm. 2014: 141747.

18. Brückner D, Hafner F-T, Li V, Schmeck C, Telser J, Vakalopoulos A, et al. 2005. Dibenzodioxocinones-A new class of CETP inhibitors. Bioorg. Med. Chem. Lett. 15: 3611-3614.

19. Gao J-M, Yang S-X, Qin J-C. 2013. Azaphilonoids: chemistry and biology. Chem. Rev. 113: 4755-4811.

20. Luo X, Lin X, Tao H, Wang J, Li J, Yang B, et al. 2018. Isochromophilones A-F, cytotoxic chloroazaphilones from the marine mangrove endophytic fungus Diaporthe sp. SCSIO 41011. J. Nat. Prod. 81: 934-941.

21. Cui H, Yu J, Chen S, Ding M, Huang X, Yuan J, et al. 2017. Alkaloids from the mangrove endophytic fungus Diaporthe phaseolorum SKS019. Bioorg. Med. Chem. Lett. 27: 803-807.

22. Parisot D, Devys M, Barbier M. 1989. Notizen: 5-Deoxybostrycoidin, a new metabolite produced by the fungus Nectria haematococca (Berk. and Br.) Wr. Z. Naturforsch. B 44: 1473-1474.

23. Li G, Kusari S, Kusari P, Kayser O, Spiteller M. 2015. Endophytic Diaporthe sp. LG23 produces a potent antibacterial tetracyclic triterpenoid. J. Nat. Prod. 78: 2128-2132.

24. Sharma V, Singamaneni V, Sharma N, Kumar A, Arora D, Kushwaha M, et al. 2018. Valproic acid induces three novel cytotoxic secondary metabolites in Diaporthe sp., an endophytic fungus from Datura inoxia Mill. Bioorg. Med. Chem. Lett. 28: $2217-2221$.

25. Ito A, Kumagai I, Maruyama M, Maeda H, Tonouchi A, Nehira T, et al. 2016. Homopetasinic acid isolated from Diaporthe sp. strain 1308-05. Tetrahedron Lett. 57: 1117-1119.

26. Schlob S, Hackl T, Herz C, Lamy E, Koch M, Rohn S, et al. 2017. Detection of a toxic methylated derivative of phomopsin A produced by the legume-infesting fungus Diaporthe toxica. J. Nat. Prod. 80: 1930-1934.

27. Cui H, Lin Y, Luo M, Lu Y, Huang X, She Z. 2017. Diaporisoindoles A-C: three isoprenylisoindole alkaloid derivatives from the mangrove endophytic fungus Diaporthe sp. SYSU-HQ3. Org. Lett. 19: 5621-5624.

28. Cui H, Liu Y, Li J, Huang X, Yan T, Cao W, et al. 2018. Diaporindenes A-D: four unusual 2,3-dihydro-1H-indene analogues with antiinflammatory activities from the mangrove endophytic fungus Diaporthe sp. SYSU-HQ3. J. Org. Chem. 83: 11804-11813.

29. Tian W, Liao Z, Zhou M, Wang G, Wu Y, Gao S, et al. 2018. Cytoskyrin C, an unusual asymmetric bisanthraquinone with cage-like skeleton from the endophytic fungus Diaporthe sp. Fitoterapia 128: 253-257.

30. Liu Z, Zhao J, Liang X, Lv X, Li Y, Qu J, Liu Y. 2018. Dothiorelone derivatives from an endophyte Diaporthe pseudomangiferaea inhibit the activation of human lung fibroblasts MRC-5 cells. Fitoterapia 127: 7-14.

31. Gao YQ, Du ST, Xiao J, Wang DC, Han WB, Zhang Q, et al. 2020. Isolation and characterization of antifungal metabolites from the Melia azedarach-associated fungus Diaporthe eucalyptorum. J. Agric. Food Chem. 68: 2418-2425. 